\section{Pilot Study of Alternative Therapy with Minerals in Patients of Polycystic Ovary Syndrome}

\section{Abstract}

Background: Polycystic ovary syndrome (PCOS) is a common endocrine system disorder among women of reproductive age. Women with PCOS may have enlarged ovaries that contain small collections of fluid (Follicles). Alternative therapy including natural minerals has been emerging in addition to established medical and surgical treatment for PCOS. In present non-randomized, open label pilot study, we have studied safety and effectiveness of exclusive natural mineral solution with use of reserve wellness wrapping technique in PCOS patients.

Methods: A total of 20 females (10 married and 10 unmarried) who had with PCOS were enrolled in present study. Natural mineral powder used for study consist of mixture of Bentonite Clay, Magnesium Chloride, Magnesium Sulphate, Sodium Chloride and Zinc Oxide 2\%. Patients were undergone standard study procedure. Changes in hormonal level $(\mathrm{AMH}, \mathrm{LH}, \mathrm{FSH}$, Prolactin, TSH, Insulin resistance) measured on $3^{\text {rd }}$ day of menstrual cycle pre-treatment and post treatment was evaluated to assess the efficacy of study treatment. Safety was evaluated throughout study procedure by observing adverse events. Compliance to study treatment was documented on individual case report form of the patient.

Results: Study results revealed that majority of hormonal level significantly changed post treatment compared to pre-treatment. LH/FSH Ratio at pre-treatment was 2.76:1, which was reduced to $1.21: 1$ at posttreatment $(p<0.001)$. Study results shown that there was statistically significant improvement in Luteinizing Hormone (LH), Prolactin, AMH, Fasting Insulin, SGPT, post treatment from the preoperative values $(P<0.05)$. No adverse event was reported during conduct of present study.

Conclusions: Present study procedure for PCOS treatment is found safe and efficacious without any incidence of adverse events.

Keywords: Polycystic ovary syndrome, Follicles, LH/FSH ratio
Raj Soni' and Dilip Gadhavi²

1 Corllins University, Director of Reserve Wellnes USA

2 Obstetrician and Gynecologist from NHL Municipal Medical Collage, Ahmedabad, Gujarat, India

Corresponding author: Raj Soni

झ raj@reverve.in

Corllins University, Director of Reserve Wellness, USA.

Tel: +918306067890

Citation: Soni R, Gadhavi D. Pilot Study of Alternative Therapy with Minerals in Patients of Polycystic Ovary Syndrome. J Univer Surg. 2016, 4:3.

\section{Introduction}

Polycystic ovary syndrome (PCOS) is a common endocrine system disorder among women of reproductive age. Women with PCOS may have enlarged ovaries that contain small collections of fluid - cysts - located in each ovary as seen during an ultrasound exam [1]. PCOS is defined by hyperandrogenism (clinical or biochemical), chronic anovulation, and/or polycystic ovaries with the exclusion of the adrenal, ovary and pituitary disorders. It is also characterized by multiple metabolic aberrations such as insulin resistance (IR) and hyperinsulinaemia, high incidence of impaired glucose tolerance visceral obesity, inflammation and endothelial dysfunction, hypertension and dyslipidemia resulting in an increased risk for diabetes and clinical or subclinical cardiovascular disease [2]. Compromised quality of life, anxiety and depression are also observed in PCOS. PCOS is now considered as a female subtype of the metabolic syndrome and its potential health consequences are a lifelong issue [2].

Polycystic ovary syndrome (PCOS) is one of the most common endocrinological disorders in women of reproductive age, occuring in $5 \%$ to $10 \%$ of women of reproductive age [3]. In PCOS, anovulation relates to low follicle-stimulating hormone 
concentrations and the arrest of antral follicle growth in the final stages of maturation [4].

The exact cause of polycystic ovary syndrome is unknown. Early diagnosis and treatment along with weight loss may reduce the risk of long-term complications, such as type 2 diabetes and heart disease [5]. A main underlying problem with PCOS is a hormonal imbalance. In women with PCOS, the ovaries make more androgens than normal. Androgens are male hormones that females also make. High levels of these hormones affect the development and release of eggs during ovulation [5].

PCOS is caused by an imbalance in the hormones $[3,4]$. PCOS usually happens when a hormone called LH (from the pituitary gland) or levels of insulin (from the pancreas) are too high, which results in extra testosterone production by the ovary.

Medical treatment of PCOS with medications such as clomiphene citrate, tamoxifen, aromatase inhibitors, metformin, glucocorticoids, or gonadotropins or surgically by laparoscopic ovarian drilling is under practise. In vitro fertilization will remain the last option to achieve pregnancy when others fail. Chronic anovulation over a long period of time is also associated with an increased risk of endometrial hyperplasia and carcinoma, which should be seriously investigated and treated. There are androgenic symptoms that will vary from patient to patient, such as hirsutism, acne, and/or alopecia. These are troublesome presentations to the patients and require adequate treatment [4].

Alternative medicine has been emerging as one of the commonly practiced medicines for different health problems. Alternative medicines include many modalities, such as kinesiology, herbalism, homeopathy, reflexology, acupressure, acupuncture, and massage therapy. Acupuncture is the most common modality. Low-frequency EA and physical exercise improved hyperandrogenism and menstrual frequency more effectively than no intervention in women with PCOS. Low-frequency EA was superior to physical exercise and may be useful for treating hyperandrogenism and oligo/amenorrhea [4]. The benefit acupuncture seems to have for PCOS sufferers is in helping them regulate and manage their periods. However, it has also been shown to aid in weight loss and reducing headaches as well as improving patients' moods and outlooks. Women with PCOS will have needles placed along the acupuncture meridians related to the reproductive system. This will help stimulate the organs, improve blood flow to the area, and contribute to normalizing hormone levels, and promote the proper functioning of the reproductive system [5].

Neutraceutical is "a food (or part of a food) that provides medical or health benefits, including the prevention and/or treatment of a disease". Scicchitano $P$ et al. [6] reviewed that these foods can protect human body from adverse events because of the beneficial effects of some photochemical. Several studies have reported the validity of this idea in clinical practice and could be considered for patients of PCOS in addition to other treatment strategy $[6,7]$.

One more approach for natural treatment is utilization of the natural mineral base containing practically every known element of the earth and taking advantage of current technology and research, there is strong possibility to improve hormonal balance in particularly PCOS patients. The wrapping process 'contours' the body which results in better balances hormones.

In present single centric, non-randomized, open label pilot study, exclusive natural mineral solution and reserve wellness wrapping technique is combined to study in PCOS patients. Objective of present study is to evaluate the efficacy and safety of Natural mineral solution in treatment of polycystic ovary syndrome. Before planning study, all types of wrap solutions known to use in women were studied. Additional evaluation for known information with the techniques and processes for the purpose of balancing hormones were carried out.

\section{Methods}

Both married and unmarried female patients with Polycystic Ovary Syndrome (PCOS) who are willing to sign a copy of the written Informed Consent form and agree to attend all follow-up appointments were included in present study. Pregnant females and females with Phlebitis and/or Emphysema were excluded from study. Patients with allergies to metal mined from earth or any study Ingredient and who had undergone any major surgery in last 3 months were also excluded from this study. Post-natal and lactating women, patients with cancer, lupus and heart problems, epilepsy, hypertension, hypotension, skin diseases or disorders, diabetes and asthma were wrapped in study with special precautions.

Present study was conducted in accordance with the ethical principles that have their origins in the Declaration of Helsinki along with the local regulatory requirements of GCP for Clinical Research in India, Schedule Y, ICMR guidelines for Biomedical Research on Human Patients and all relevant SOPs of Study centre. Ethical approval of study documents was obtained from ethics committee in written before conduct of study, as per regulatory requirement. Details of the study such as objectives, procedures, risks, benefits, restrictions and requirements of the study was presented to all the patients in a language that the patient best comprehended. The patients were given ample time to read the ICF followed by explanation by the study personnel. All the queries of the patient were resolved before obtaining the consent. All patients were encouraged to ask questions and all the questions were fully answered. Study specific written informed consent was obtained from each patient before enrolling into the study.

A total of 20 females (10 married and 10 unmarried) who fulfilled predefined inclusion/exclusion criteria were enrolled in present study. Baseline demography of Patient age, height and weight was recorded for individual patients.

Patients were undergone study procedure and efficacy data were collected at pre study and post study treatment. Natural mineral powder used for study consist of mixture of Bentonite Clay, Magnesium Chloride, Magnesium Sulphate, Sodium Chloride and Zinc Oxide 2\%.

Study was single arm pen label study with only one treatment arm so method of assigning patients to treatment groups is not applicable. Individual patient was given prescheduled 
appointment and asked to visit clinic for study procedure which was done for approximately 1 hour and repeated every $4^{\text {th }}$ day for 1 complete menstrual cycle. Treatment was recommended to be taken after 2 hours of lunch. Patients were advised not to apply any type of body cream, lotion, deodorant or perfume, bath soap, and body powder while duration of study. Patient had to drink at least 12 to 14 glass of water after treatment every day.

Total of approximately 30 nos. of bandages were soaked in specialized water heating tank along with $1 \mathrm{~kg}$ mineral powder in 4.5 litres of water at least for $60 \mathrm{~min}$. At every $15 \mathrm{~min}$, bandages were turned around in water heating tank so that all minerals were getting absorbed by elasticated cotton bandages. Participants were wrapped from neck to toe, from legs upwards and then covered with vinyl suit, to maintain body temperature. The patients were remained in lied down position for one hour.

After one hour, vinyl suit is removed and after unwrapping the patient wipe the body. Patients were not advised to take even shower after treatment.

Compliance to study treatment was documented on individual case report form of the patient.

$5 \mathrm{ml}$ blood was collected on $3^{\text {rd }}$ day of menstrual cycle to investigate hormonal level in individual patient at baseline (before treatment) and post treatment ( $3^{\text {rd }}$ day of next menstrual cycle). Laboratory investigation for hormones $\mathrm{AMH}, \mathrm{LH}, \mathrm{FSH}$, Prolactin, TSH, Insulin resistance was performed for efficacy evaluation. Efficacy was evaluated by Improvement in hormone balance $(\mathrm{AMH}, \mathrm{LH}, \mathrm{FSH}, \mathrm{LH} / \mathrm{FSH}$ ratio, Prolactin, TSH and Insulin resistance). Safety was evaluated throughout study procedure as well as at end of study. Safety was evaluated by analyzing number of adverse events and patients with adverse events.

Sample size was not formally calculated for present study, considering pilot study. Statistical analysis was performed using SPSS software. Frequency (N), Mean (SD), Minimum, Maximum values were reported for continuous variables. Frequency and percentage were displayed for categorical variables. Statistical significance for efficacy variables were evaluated between pre study mean value and post study mean by paired $t$ test.

There were no protocol deviations reported during conduct of study. None of the patients was discontinued from present study.

\section{Results}

All enrolled (twenty) patients had completed the study and were included for safety and efficacy analysis. Proportion of married and unmarried patients were equal (50\%) in present study. Mean age of patients enrolled in present study was $26.85 \pm 4.75$ years (range 19-35 years).

Minimum-maximum of age (in years), height (in $\mathrm{cm}$ ), weight (in $\mathrm{kg}$ ) and BMI at baseline is presented in Table 1.

\section{Efficacy Results}

Changes in hormonal level measured on $3^{\text {rd }}$ day of menstrual cycle pre- and post-treatment was evaluated to assess the efficacy of study treatment.
Table 1: Baseline Demographic characteristics ( $N=20)$.

\begin{tabular}{|c|c|c|c|c|}
\hline Parameters & Age (In Years) & Height $(\mathrm{In} \mathrm{cm})$ & Weight $(\mathrm{ln} \mathrm{Kg})$ & BMI \\
\hline Mean & 26.85 & 156 & 70.44 & 28.9 \\
\hline SD & 4.75 & 3.55 & 4.19 & - \\
\hline Min & 19 & 150 & 64.5 & - \\
\hline Max & 35 & 163 & 79.2 & - \\
\hline
\end{tabular}

Study results revealed that majority of hormonal level significantly changed post treatment compared to pre-treatment. There was statistically significant improvement in Luteinizing Hormone (LH), Prolactin, Anti-Mullerian Hormone (AMH), Fasting Insulin, SGPT and $\mathrm{LH} / \mathrm{FSH}$ ratio, post-treatment from the preoperative values $(\mathrm{P}<0.05)$.

Baseline mean value of FSH was $4.18 \pm 0.76$ which was normal and remained almost unchanged (mean value $5.20 \pm 0.94$ ) posttreatment. P-value provided by statistical comparison of $\mathrm{FSH}$ level by paired t-test is 0.131 which revealed that there is no statistically significant difference at post treatment.

Baseline mean value of $\mathrm{LH}$ was $11.52 \pm 2.16$ which was normal and persisted in normal range (mean value $4.27 \pm 0.65$ ) posttreatment. P-value provided by statistical comparison of LH level by paired t-test indicated highly statistically significant difference at post treatment in LH level. LH/FSH Ratio at pre-treatment was 2.76:1 which was high compared to healthy female with usual hormonal level, which was reduced positively to $1.21: 1$ at post-treatment. This reduction is significant clinically as well as statistically $(p<0.001)$.

Baseline mean value of TSH was $3.63 \pm 0.65$ which was normal and stable (mean value $3.62 \pm 0.64$ ) at post-treatment. There is no statistically significant difference at post treatment in TSH value $(p=0.207)$ compared to pre-treatment.

Initial prolactin level pre-treatment was little higher (mean value $36.36 \pm 1.94)$ at baseline was reduced to normal level with mean value $29.35 \pm 1.32$ at post-treatment $(p<0.001)$. $\mathrm{AMH}$ level was observed normal (mean $6.79 \pm 0.18$ ) at post-treatment which was found higher than normal at baseline (mean value $10.84 \pm 1.25$, $\mathrm{p}<0.001$ ).

Fasting Insulin mean value was normal at pre-treatment as well as post-treatment and stable $(p=0.018)$.

Initial SGPT level pre-treatment was little higher (mean value $35.83 \pm 1.41$ ) at baseline was reduced to normal level with mean value $29.40 \pm 1.68$ at post-treatment $(p<0.001)$.

Comparison of pre-treatment and post-treatment mean value with SD is presented in Table 2. Pre-treatment and post-treatment mean value of each hormonal level is depicted in Figure 1.

Study results had shown that all hormonal levels had reduced little or more at post treatment except FSH level. LH/FSH ratio was also significantly reduced. Mean change of hormonal level at post treatment compared to pre-treatment is presented in Table 3.

Treatment Compliance was evaluated and documented by record of menstruation date and clinic treatment dates in CRF of each patient. Most of patients received seven $(n=12)$ or eight times $(n=6)$ study treatment. Details of patients receiving respective number of treatment are provided in Table 4. 
Table 2: Comparison of pre-treatment and post treatment hormonal level.

\begin{tabular}{|c|c|c|c|c|c|c|c|c|c|c|c|}
\hline \multirow{2}{*}{ Parameter } & \multirow{2}{*}{ Range } & \multirow{2}{*}{ Unit } & \multicolumn{4}{|c|}{ Pre treatment Values } & \multicolumn{4}{|c|}{ Post treatment Values } & \multirow{2}{*}{ P-Value } \\
\hline & & & Mean & S.D. & Min & Max & Mean & S.D & Min & Max & \\
\hline FSH & 4.00 to 13.0 & $\mathrm{mlU} / \mathrm{ml}$ & 4.18 & 0.76 & 2.91 & 5.6 & 4.27 & 0.65 & 3.15 & 5.6 & 0.131 \\
\hline LH & 1.90 to 12.5 & $\mathrm{mlU} / \mathrm{ml}$ & 11.52 & 2.16 & 8.18 & 16.75 & 5.2 & 0.94 & 3.75 & 7.1 & $<.0001$ \\
\hline Prolactin & 2.8 to 29.2 & $\mathrm{ng} / \mathrm{ml}$ & 36.36 & 1.94 & 31.17 & 39.1 & 29.35 & 1.32 & 27.61 & 31.25 & $<0.001$ \\
\hline TSH & 0.35 to 5.5 & microlU/I & 3.63 & 0.65 & 2.75 & 4.85 & 3.62 & 0.64 & 2.75 & 4.75 & 0.207 \\
\hline $\mathrm{AMH}$ & 4.00 to 6.79 & $\mathrm{ng} / \mathrm{ml}$ & 10.84 & 1.25 & 8.95 & 12.81 & 6.79 & 0.18 & 6.26 & 7.02 & $<0.001$ \\
\hline Fasting Insulin & 2.6 to 37.6 & $\mathrm{mU} / \mathrm{I}$ & 15.77 & 1.94 & 11.6 & 18.2 & 15.32 & 1.53 & 12.1 & 17.8 & 0.018 \\
\hline S.G.P.T. & 0 to 31 & $\mathrm{U} / \mathrm{L}$ & 35.83 & 1.41 & 33.2 & 38.1 & 29.4 & 1.68 & 26.7 & 32.2 & $<0.001$ \\
\hline LH/FSH Ratio & 01:01 & & 2.76 & 0.18 & 2.47 & 3.1 & 1.21 & 0.09 & 1.1 & 1.49 & $<0.001$ \\
\hline
\end{tabular}

\section{- Pre treatment Values Mean}

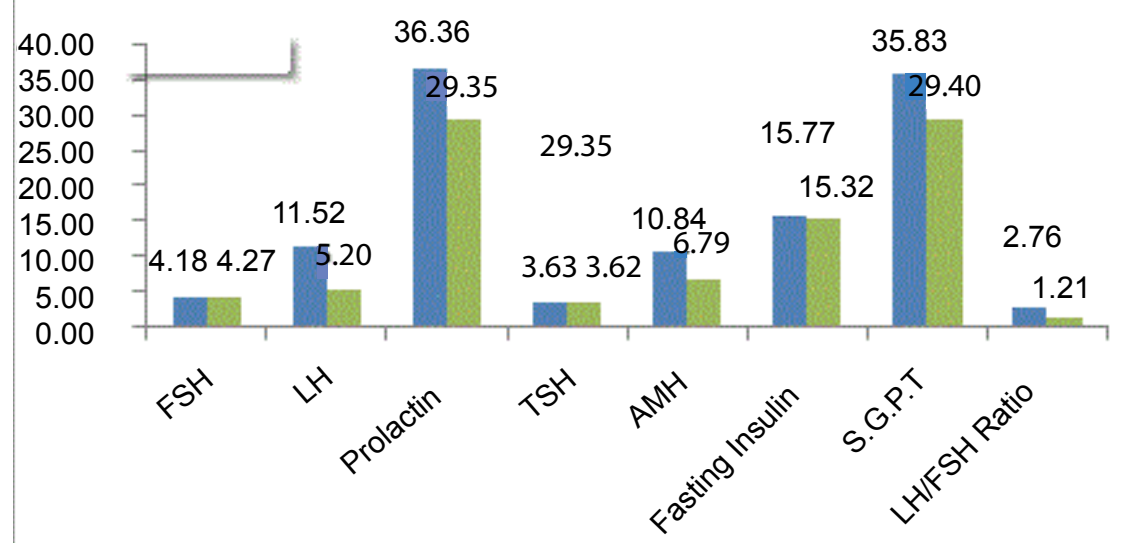

Figure 1 Comparison of pre-treatment and post treatment hormonal level.

Study results favoured effectiveness of mineral therapy in treatment of patients with PCOS.

\section{Safety Results}

All 20 patients were exposed to study treatment and were included in safety analysis. No adverse event was reported during conduct of present study. Present study procedure for PCOS treatment is found safe and well tolerable without any incidence of adverse events.

\section{Discussion}

The present study was performed to evaluate safety and efficacy of alternative therapy with minerals in patients of PCOS. Exclusive natural mineral solution and reserve wellness wrapping technique is combined to study in PCOS patients. Before planning study, all types of wrap solutions known to use in women were studied. Additional evaluation for known information with the techniques and processes for the purpose of balancing hormones were carried out.

Mineral solution base used in present study is natural minerals. Individual minerals have shown advantageous role in PCOS patient as a natural therapy but specific combination of minerals with well-designed specific technique was not studied till date $[2,7]$. Present study was carried out on hypothesis of utilizing the natural mineral base containing practically every known element of the earth and taking advantage of current technology and research, there is strong possibility to improve hormonal balance in particularly PCOS patients.

Hormones play vital role in female reproduction system. Improvement in hormonal balance may beneficial in many gynaecological diseases including Polycystic ovary syndrome (PCOS). There was statistically significant improvement in Luteinizing Hormone (LH), Prolactin, AMH, Fasting Insulin, SGPT and $\mathrm{LH} / \mathrm{FSH}$ ratio, post-treatment from the preoperative values $(P<0.05)$.

We have not placed any patients on diet change or any life style modifications. So, weight and BMI was not studied at end of treatment. LH/FSH Ratio at pre-treatment was 2.76:1 which was high compared to healthy female with usual hormonal level, which was reduced positively to $1.21: 1$ at post-treatment. This reduction is significant statistically $(<0.001)$. In healthy females, generally this ratio may be near to $1: 1$, so study results are approaching towards positive clinical aspects.

Any complications or adverse events were not reported in present report denoted the safety of this procedure.

Both married and unmarried females were included in present study equally. Sample size of 10 married and 10 unmarried females were quite less and insufficient to establish proof of concept for efficacy and safety. 
Table 3: Post treatment reduction in hormonal level compare to pretreatment (Efficacy Population).

\begin{tabular}{|c|c|c|}
\hline & \multicolumn{2}{|c|}{ Reduction at post treatment } \\
\hline FSH & 0.09 & S.D. \\
\hline LH & -6.32 & -0.11 \\
\hline Prolactin & -7.01 & -1.21 \\
\hline TSH & -0.01 & -0.62 \\
\hline AMH & -4.05 & -0.01 \\
\hline Fasting Insulin & -0.46 & -1.07 \\
\hline S.G.P.T & -6.43 & -0.4 \\
\hline LH/FSH Ratio & -1.55 & 0.27 \\
\hline
\end{tabular}

Table 4: Patients received treatment.

\begin{tabular}{|c|c|}
\hline Number of treatment procedure & Number of patients - N (\%) \\
\hline No. of Patients receive 6 times & $1(5 \%)$ \\
\hline No. of Patients receive 7 times & $12(60 \%)$ \\
\hline No. of Patients receive 8 times & $6(30 \%)$ \\
\hline No. of Patients receive 9 times & $1(5 \%)$ \\
\hline
\end{tabular}

In conclusion, use of natural minerals with specific study procedure is a safe measure with promising primary study results for polycystic ovary syndrome (PCOS) for treatment of hirsutism, at least in the short term as demonstrated by this study. This method is technically simpler, cheaper and less invasive than other therapies proposed for polycystic ovary syndrome (PCOS). Mineral treatment may also be helpful for treatment of infertility. Further studies with more patients and longer follow-up to verify the stability of the induced effect are recommended to investigate further efficacy including fertility rate and pregnancy in PCOS patient population.

\section{Limitation}

Controlled clinical trials generally carried out as randomized allocation of treatment, either with active comparator or placebo, by equalising some factors that have not been accounted for in the experimental design e.g., a group of people with a health condition, different from the disease under study. In present study, lack of randomization may have bias, which is suspected to affect treatment efficacy and limitation of study. One another major limitation is lack of statistical consideration of sample size. Data generated from small group of patients could not reliable as results while larger exposure in patients may be different due to patient variability. Ideally, multivariate regression analysis should be performed in order to evaluate the role of confounding factors on results but less number of sample sizes is limiting factor for such post-hoc analysis. As researcher wanted to conduct this study as pilot basis, future studies with randomized controlled and ideal study design are recommended to confirm outcomes of present study.

\section{Acknowledgements}

We would like to thank Mr. Suresh Bhatol and Ethi trials Clinical Research Pvt. Ltd. for valuable support in writing manuscript.

\section{Declarations}

Funding: All funding for present study was provided by Reserve Wellness.

Conflict of interest: No conflict of Interests.

Ethical approval: Ethical approval for conduct of study was received from Ethics Committee before conduct of study. 


\section{References}

1 Mayo Clinic (2016) Diseases and Conditions Polycystic ovary syndrome (PCOS).

2 Bargiota A, Diamanti-Kandarakis E (2012) The effects of old, new and emerging medicines on metabolic aberrations in PCOS. Ther Adv Endocrinol Metab 3: 27-47.

3 Turan V, Sezer ED, Zeybek B, Sendag F (2015) Infertility and the presence of insulin resistance are associated with increased oxidative stress in young, non-obese Turkish women with polycystic ovary syndrome. J Pediatr Adolesc Gynecol 28: 119-123.
4 Jedel E, Labrie F, Odén A, Holm G, Nilsson L, et al. (2011) Impact of electro-acupuncture and physical exercise on hyperandrogenism and oligo/amenorrhea in women with polycystic ovary syndrome: a randomized controlled trial. Am J Physiol Endocrinol Metab 300: E37-E45.

5 Badawy A, Abubaker E (2011) Treatment options for polycystic ovary syndrome. Int J Womens Health 3: 25-35.

6 Scicchitano P, Cameli M, Maiello M, Modesti PA, Muiesan ML, et al. (2014) Nutraceuticals and dyslipidaemia: beyond the common therapeutics. J Funct Foods 6: 11-32.

7 http://womenshealth.gov/publications/our-publications/factsheet/polycystic-ovary-syndrome.html 